\title{
Stress Estimation of ECG Signal by using DWT
}

\author{
Purushottam Pratap Singh, A. K Wadhwani and S. Wadhwani \\ Department of Electrical Engineering \\ Madhav Institute of Science \& Technology, Gwalior, India \\ ppratap2010@gmail.com
}

\begin{abstract}
Identification and prediction of stress using existing data processing methodologies are incompetent to predict the stress either in real time or laboratory based experiments. The main aim of this work is to classify the stress. The appropriate signal processing methodology, (i) to analyze the Electrocardiogram (ECG) signal for normal and stress condition and (ii) to derive the optimum features from a set of statistical features over frequency band. This paper represent method to find out stress condition. Electrocardiogram, were recorded continuously while drivers followed a set route through open roads in the greater Boston area. Stress levels are created by driving into different condition. By driving in city, highway and rest condition. We used physionet data base 'Stress Recognition in Automobile Drivers' for ECG signal.ECG signal is used to analysis the stress. Parameters( RMS, Max ,Std ,kurtosis , min ,mean ,variance)are used for analysis by using discrete wavelet transform by using 'db6' mother wavelet function . Entropy Correlation, Skew ness, median, crest factor are not giving the appropriate changes from normal condition to stressed condition so these parameters are not considered. Artificial neural network was used for decision making you are in stress or not.
\end{abstract}

Keywords: electrocardiogram, automobile driver, stress, discrete wavelet transform, artificial neural network

\section{Introduction}

Psychosocial factors influence the coronary artery diseases [1]. Human encountered the stress due to psychosocial factors like working environment, family issues, job, traffic, more physical and mental demands etc. Management of stress practiced is usually very among the people using suitable stress level reduction methods. The quantization's of stress also not straight forward using scientific tool. Recent days, researchers are working on identifying and classifying stress through physiological signals for developing efficient stress releivement. Numerous works are developed to estimate the stress through questionnaire approach [2-4], biochemical sample analysis [5, 6] and physiological signal analysis [7-9]. As a result, the physiological signal approach is more successful than other methods. Effect of stress on body can be easily analyzed using physiological signals. Several physiological signals are used to measure the stress such as ECG, Heart Rate Variability (HRV), Electromyogram (EMG), Galvanic Skin Response (GSR), Skin Temperature (ST), Respiration Rate (RR), and Blood Pressure (BP) [6-8]. To investigate the physiological signals under stress, several real time and a laboratory task were considered by several researchers $[5,6]$. Stress of the car drives has been used here during different driving conditions [7].

So for, various researchers are identified the stress through various biochemical (blood, urine, and saliva etc.) and physiological parameters [12. 13]. Among the several physiological signals, the HRV signal is mostly used to identify the stress. In addition, HRV signal is not only used to study stimuli based stress measurement and also used in 
heart related diseases like Coronary Artery Disease (CAD) and myocardial ischemia due to stress [1]. Most of the researchers have investigated about the characteristic changes of high frequency (HF) and low frequency (LF) bands from HRV signals for stress identification and its level measurements [7,9]. Only very few researchers have discussed the effect of stress in the ECG signals for stress assessment. Interestingly, recent studies reported that ECG signal has been progressively used to identify the Autonomic Nervous Activity (ANS) of emotion and stress identification [14, 15].

In this study, we aim to classify the stress using the ECG signal by analyzing several statistical features. Feature extraction is performed nonlinear feature extraction method namely Discrete Wavelet Transform (DWT) using "db6" mother wavelet function. Seven different statistical features were computed and decision taken by Artificial Neural Network (ANN).

\section{Methods and Materials}

\section{A. Driving Protocol}

The driving protocol consisted of path of open road. Generally stressful condition is not created in open condition. Route is planed in such a way that driver could be go into different traffic conditions. These different conditions create different stressful condition. Two 15 minute rest period is given at first and last of driving time. In rest condition driver sat on the chair and closed his eyes at this condition we record the ECG signal. This first time period is considered as a normal time period. After that time period driver goes into city .In the city traffic is more than open road, so this create stressful condition.

\section{B. Subjects and Data Acquisition}

To participate in the experiment drivers should be valid driver license and they are to consent for recording of ECG signal during driving. Before starting the driving a map of route was given to the drivers and some instruction was given such as (i) speed should be consistent and (ii) not to listen the radio because if you are in stress condition recording of ECG is going on. If you listen the radio it make you relax and quality of ECG signal can be reduced. During the drive, an observer accompanied the driver in the car .He gives the answer of any question of driver and monitor the quality of the signal that is recorded.

\section{Data Processing}

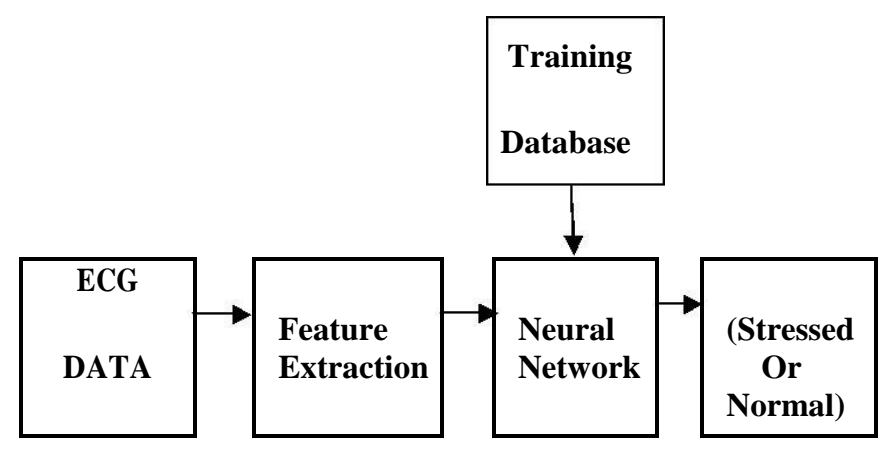

Figure 1. Block Diagram of Stress Test Process

\section{A. Electrocardiogram.}

It is the electrical activity of the heart. Stress increase the heart rate, blood pressure, so the electrocardiogram is changed. By analysis of the ECG we can find out the impact 
of the stress on the body. Previously many studies show that heart rate variability is main dominant for analysis of stress. Due to the stress heart rate is badly affected .Generally it is shown that important information is occur in the below $100 \mathrm{~Hz}$ signal. We used frequency band for analysis of ECG signal. Here we decomposed the signal upto $3^{\text {rd }}$ level by using discrete wavelet transform. We used 'db6' mother wavelet function for analysis of the signal. Artificial neural network is used for decision making whether it is in stressed condition or normal condition.

\section{B. Feature Extraction.}

There are so many technique for analysis of signal in signal processing area, Such as Fourier Transform (FT) and Short Term Fourier Transform (STFT), Empirical Mode Decomposition (EMD) and Discrete Wavelet Transform (DWT).Generally discrete wavelet transform is used for analysis of signal in case of stress and emotion. The efficient feature extraction methods only capable to analyze nonlinear signal without loss of information. In this work we used ' $\mathrm{db} 6$ ' mother wavelet function was chosen to identify optimum features for the several statistical features of frequency band. Firstly ECG signal was passed through the discrete wavelet transform.DWT break down the signal into two parts by using 'db6' wavelet transform. We can say that signal passed through the filters one is low pass filter and other Is high pass filter. Component of low pass filter is called approximate coefficient and component of high pass filter is called details coefficient. This approximation coefficient (CAL) is again decomposed until 3rd level in order to obtain the required frequency of the band.

\section{Feature Computation and Normalization}

Maximum, mean, min, standard deviation (Std. dev), RMS, kurtosis, variance features were taken to measure the stress levels changes of ECG signals. We use frequency band of $3^{\text {rd }}$ level for calculating the following parameters. We take one person data .Total ECG data was 66 minute duration. First 15 min was considered as normal data and remaining data was considered as a stressed data. We take 10 epochs of equal duration of normal data and also 10 epochs of equal duration of stressed data. We calculated these parameters for each epochs. So we get matrix of size $7 * 20$. These database are used for training the neural network. $70 \%$ of the epochs are used for training, $15 \%$ of epochs for validation and $15 \%$ epochs for testing. We make two matrix one was testing matrix and other was target matrix. Size was target matrix is $1 * 20$. We take 0 for normal person and 1 for stressed person.

\section{Artificial Neural Network}

Neural network has architecture inspired by human brain. It is a massively parallel distributed processing system, which is made of highly interconnected neural computing elements. Theses neural computing elements have ability to learn. So these neural networks are acquire knowledge and make us available to use this knowledge. The neural network highly interconnected network of a large number of processing elements called neurons.

Neural network learns by example. So neural networks can be trained by providing known examples of a problem than once if appropriately get trained then this network can be put to effective use in solving unknown and untrained instances of problem. In this work we use neural network toolbox for train the neural network. We take 20 epochs of same length for training the network. Here 10 epochs of normal person and 10 epochs of stressed person are taken. we calculated 7 parameters( RMS , max, standard deviation, kurtosis, min ,max, variance) of each epoch. So total $20 * 7=140$ parameters vectors. $70 \%$ epochs are taken for training and $15 \%$ epochs for validation and testing. 


\section{Result and Discussion}

Table 1. Parameters of each Epoch for Stressed Condition

\begin{tabular}{|c|c|c|c|c|c|c|c|c|c|c|}
\hline Parameters & E1 & E2 & E3 & E4 & E5 & E6 & E7 & E8 & E9 & E10 \\
\hline RMS & $\mathbf{0 . 0 1 3 4}$ & $\mathbf{0 . 0 1 4 2}$ & $\mathbf{0 . 0 1 4 2}$ & $\mathbf{0 . 0 1 3 9}$ & $\mathbf{0 . 0 1 3 6}$ & $\mathbf{0 . 0 1 4 5}$ & $\mathbf{0 . 0 1 3 4}$ & $\mathbf{0 . 0 1 6 2}$ & $\mathbf{0 . 0 1 3 3}$ & $\mathbf{0 . 0 1 3}$ \\
\hline max & $\mathbf{0 . 0 4 4 2}$ & $\mathbf{0 . 0 4 2 4}$ & $\mathbf{0 . 0 4 6 1}$ & $\mathbf{0 . 0 2 9 7}$ & $\mathbf{0 . 0 2 5 0}$ & $\mathbf{0 . 0 2 8 6}$ & $\mathbf{0 . 0 2 4 6}$ & $\mathbf{0 . 0 7 2}$ & $\mathbf{0 . 0 2 5 4}$ & $\mathbf{0 . 0 2 6}$ \\
\hline Std & $\mathbf{0 . 0 1 3 4}$ & $\mathbf{0 . 0 1 4 2}$ & $\mathbf{0 . 0 1 4 2}$ & $\mathbf{0 . 0 1 3 9}$ & $\mathbf{0 . 0 1 3 7}$ & $\mathbf{0 . 0 1 4}$ & $\mathbf{0 . 0 1 3 4}$ & $\mathbf{0 . 0 1 6 2}$ & $\mathbf{0 . 0 1 3 4}$ & $\mathbf{0 . 0 1 3 7}$ \\
\hline kurtosis & $\mathbf{0 . 0 0 0 2}$ & $\mathbf{0 . 0 0 0 2}$ & $\mathbf{0 . 0 0 0 2}$ & $\mathbf{0 . 0 0 0 1 9 8}$ & $\mathbf{0 . 0 0 0 1 9 2}$ & $\mathbf{0 . 0 0 0 2 1 1}$ & $\mathbf{0 . 0 0 0 1 9 2}$ & $\mathbf{0 . 0 0 0 3 6}$ & $\mathbf{0 . 0 0 0 2}$ & $\mathbf{0 . 0 0 0 1 9 1}$ \\
\hline min & $\mathbf{- 0 . 0 2 3}$ & $\mathbf{0 . 0 2 7 9}$ & $\mathbf{- 0 . 0 3 3}$ & $\mathbf{- 0 . 0 3 0 5}$ & $\mathbf{- 0 . 0 2 7 5}$ & $\mathbf{- 0 . 0 3 5 8}$ & $\mathbf{- 0 . 0 3 0 8}$ & $\mathbf{- 0 . 0 4 6 0}$ & $-\mathbf{0 . 0 3 2}$ & $\mathbf{- 0 . 0 3 1}$ \\
\hline mean & $\mathbf{0 . 0 0 0 3}$ & $\mathbf{- 1 . 1 3}$ & 4.367 & $\mathbf{0 . 0 0 0 2 0}$ & $\mathbf{0 . 0 0 0 4}$ & $\mathbf{0 . 0 0 0 2 9 0}$ & $\mathbf{- 0 . 0 0 0 3 8}$ & $\mathbf{0 . 0 0 1 1}$ & $\mathbf{0 . 0 0 0 7 3}$ & $\mathbf{0 . 0 0 0 5 9}$ \\
\hline variance & $\mathbf{1 . 8 1 2 7}$ & $\mathbf{2 . 0 3 0}$ & $\mathbf{2 . 0 4 1}$ & $\mathbf{1 . 9 5}$ & $\mathbf{1 . 8 3}$ & $\mathbf{2 . 1 1 5}$ & $\mathbf{1 . 8 1 3}$ & $\mathbf{2 . 6 3 6}$ & $\mathbf{1 . 7 9}$ & $\mathbf{1 . 8 8}$ \\
\hline
\end{tabular}

Table 2. Parameters of each Epoch for Normal Condition

\begin{tabular}{|c|c|c|c|c|c|c|c|c|c|c|}
\hline Parameters & $\mathrm{E} 1$ & $\mathrm{E} 2$ & $\mathrm{E} 3$ & $\mathrm{E} 4$ & $\mathrm{E} 5$ & $\mathrm{E} 6$ & $\mathrm{E} 7$ & $\mathrm{E} 8$ & $\mathrm{E} 9$ & $\mathrm{E} 10$ \\
\hline RMS & 0.0153 & 0.0145 & 0.0133 & 0.0126 & 0.0131 & 0.0123 & 0.0122 & 0.0139 & 0.0221 & 0.0132 \\
\hline max & 0.0756 & 0.0315 & 0.025 & 0.0241 & 0.036 & 0.0246 & 0.0235 & 0.0279 & 0.0548 & 0.0245 \\
\hline Std & 0.0153 & 0.0146 & 0.0134 & 0.0127 & 0.0131 & 0.0124 & 0.0122 & 0.0139 & 0.0221 & 0.0133 \\
\hline kurtosis & 0.0005 & 0.0002 & 0.0002 & 0.0002 & 0.0002 & 0.0002 & 0.0002 & 0.0002 & 0.001 & 0.0002 \\
\hline min & $-\mathbf{- 0 . 0 5 1 8}$ & $-\mathbf{- 0 . 0 3 3 4}$ & -0.0293 & -0.0236 & -0.0235 & -0.0238 & -0.0233 & -0.0345 & -0.1324 & -0.0288 \\
\hline mean & 0.0002 & 0.0007 & 0.0002 & 0 & 0.0003 & 0 & 0.0002 & -0.0008 & -0.0001 & 0 \\
\hline variance & 2.3474 & 2.121 & 1.788 & 1.6084 & 1.7119 & 1.529 & 1.4928 & 1.9395 & 4.8997 & 1.7623 \\
\hline
\end{tabular}

By analysis of parameters of normal and stress condition we can take decision you are in stress or normal condition. Table 1 and Table 2 show the value of parameters of stress and normal condition of each epoch. These parameters (RMS, max ,std, kurtosis, min, mean ,variance) shows the changes in value from normal condition to stressed condition. The parameters ( Entropy, correlation, skewness, median, crest factor, mean absolute variation, median absolute variation, max norm) was not showing the changes from normal condition to stressed condition. So that these parameters are not considered .Artificial neural network was used to take decision weather person is in normal state and stress condition. If the person is in normal stress it gives value 0 and if the person is in stress condition it gives value 1. The parameters of unknown ECG signal is compared with database by using neural network and take appropriate decision.

\section{Conclusion}

In this study we show a method to find out you are in stress condition or normal condition. We used ECG signal of drivers in stressed condition. For the ECG database we used physionet database 'Stress Recognition in Automobile Drivers'. In this database both normal and stressed condition ECG signal is given. We calculate appropriate frequency band for maximum information and calculate parameters by using discrete wavelet transform 'db6' mother wavelet function. Some parameter shows changes in value from normal state to stressed state some are not. We take 10 epochs of equal duration for both normal and stressed condition ECG signal and calculate the parameters for each epoch. We get a matrix of value of parameter of size $7 * 20$. Decision was taken by neural network. This neural network is trained by parameters of normal value and stressed value. We take 0 for normal condition and 1 for stressed condition.

\section{References}

[1] P. C. Strike and A. Steptoe, "Systematic review of mental stress-induced myocardial ischaemia", European Heart Journal, vol. 24, (2003), pp. 690-703. 
[2] T. H. Holmes and R. H. Rahe, "The social readjustment rating scale", Journal of Psychosomatic Research, vol. 11, (1967), pp. 213218.

[3] C. Kirschbaum, "The 'Trier Social Stress Test'- A Tool for Investigating Psychobiological Stress Responses in a Laboratory Setting", Neuropsychobiology, vol. 28, (1993), pp. 76-81.

[4] P. Karthikeyan, M. Murugappan and S. Yaacob, "A Study on Mental Arithmetic Task based Human Stress Level Classification Using Discrete Wavelet Transform", IEEE Conference on Sustainable Utilization and Development in Engineering and Technology (STUDENT), (2012), pp.77,81, (2012) October 6-9. 
International Journal of Bio-Science and Bio-Technology

Vol.7, No.5 (2015) 